\title{
Evaluation of Neutrophil-Lymphocyte Ratio as a Prognostic Factor in Cervical Intraepithelial Neoplasia Recurrence
}

\author{
Farah Farzaneh, Nafiseh Faghih*, Maryam Sadat Hosseini, Maliheh Arab, \\ Tahereh Ashrafganjoei, Atyeh Bahman
}

\begin{abstract}
Background: Immune system status is a factor related to cervical intraepithelial neoplasia (CIN) recurrence. neutrophil-lymphocyte ratio (NLR) is a useful factor in assessing the immune status. The aim of this study was to evaluate the prognostic value of NLR factor for CIN recurrence in patient who underwent excisional procedure and its relationship with recurrence free survival (RFS). Methods: In this historical cohort study, a population of 307 patients with CIN (confirmed by excisional pathology) from 2009 to 2017 were selected. NLR and hematologic factors were measured before surgery and the follow-up records of these patient were analyzed. The recurrence rate and RFS were assessed during the follow-up phase. Results: The NLR cut-off point of 1.9 was determined using the Youden Index. NLR $<1.9$ (low NLR) and NLR $\geq 1.9$ (high NLR) were observed among $68.1 \%$ and $39.1 \%$ of patients, respectively. Univariate analysis showed that higher NLR values $(\mathrm{P}<0.001)$, absolute neutrophilic counts (ANC) $(\mathrm{P}<0.001)$ and platelet lymphocytic ratios (PLR) $(\mathrm{P}=0.002)$ were significantly associated with reduction in RFS. The results of Cox regression showed that removing more tissue during excision $(\mathrm{HR}=0.325 ; 95 \% \mathrm{CI}(0.936-0.136)$ significantly reduced the hazard of recurrence, higher NLR $(\mathrm{HR}=4.55 ; 95 \% \mathrm{CI})(1.97-10.51)$ and white blood cell (WBC) count levels (HR $=1.27 ; 95 \%$ CI, 1.04-1.55), significantly decreased RFS, but PLR and ANC associated with RFS were not confirmed by Cox regression. Conclusion: NLR and total WBC count might be prognostic factors involved in the prediction of recurrence and RFS in CIN patient underwent excisional procedure. To confirm these results, more prospective studies with larger sample sizes are needed.
\end{abstract}

Keywords: Cervical intraepithelial neoplasia- recurrence- neutrophils- lymphocytes- blood platelets- conization

Asian Pac J Cancer Prev, 20 (8), 2365-2372

\section{Introduction}

The concept of preinvasive cervical lesion was introduced in 1947 which is a type of detectable epithelial change that has an invasive feature. Preinvasive cervical lesion is limited to epithelial and if left untreated, may progress to advanced form of cervical cancer (Garcia et al., 2012). The incidence of cervical cancer as one of the most common gynecological cancers has been increasing in recent decades and cervical intra-epithelial neoplasia (CIN) is known as one of the precursors to cervical cancer (Chen et al., 2018). Generally, CIN is divided into (i) CIN I which is equivalent to mild dysplasia, (ii) CIN II which is equivalent to moderate dysplasia, and (iii) CIN III which is equivalent to severe dysplasia or in-situ carcinoma (Rock and Jones, 2016). The risk of cervical cancer development depends on the severity and extent of CIN lesions (Arbyn et al., 2017). About one-third of women with untreated CIN III progress to advanced form of cervical cancer (Arbyn et al., 2017).
Large loop excision of transformation zone (LLETZ), loop electrosurgical excision procedure (LEEP), laser conization and cold-knife conization (CKC) are the most common treatment modalities in $\mathrm{CIN}$ excisional procedure (Martin-Hirsch et al., 1999; Arbyn et al., 2017). Excision or ablation reduces the risk of invasive cervical cancer in women with CIN by as much as $95 \%$ after 8 years of treatment. Most cases of the recurrence occur in the first two years, but it may occur 20 years later (Wright, 2017). There is a limited number of factors that can be used to predict the rate of CIN recurrence. Evidence suggests that marginal involvement of sample, CIN histologic grading, glandular involvement, persistent human papillomatous virus (HPV) infection, age, immune status, and immune system deficiency are important issues (Lodi et al., 2011).

Inflammation and cancer are two processes with a reflexive relationship. Understanding this relationship dates back to the $19^{\text {th }}$ century (Bottazzi et al., 2018). Leukocytosis and neutrophilia are the most common systemic changes observed in solid tumors (Pesic and 
Greten, 2016). These hematological changes are clearly related to the level of disease progression, and ultimately, to the prognosis of disease (Pesic and Greten, 2016). An unresolved chronic inflammation is found in all stages of cancer progression. About $20 \%$ of cancer cases are associated with an unresolved chronic inflammation caused by bacterial or viral infections or environmental stimuli such as tobacco smoking or autoimmune diseases (Pesic and Greten, 2016). For instance, HPV increases the production of interleukin 6 and 8 in cellular environment, and these two cytokines are associated with CIN II and CIN III progression to malignancy in $80 \%$ of cases of cancer (Wright, 2014). Neutrophils and lymphocytes of peripheral blood change in response to systemic inflammation (Ashrafganjoei et al., 2016). The immune response also causes changes in the level of WBCs in the bloodstream (Walsh et al., 2005). Neutrophils migration is the most important change in the immune response in any organ which can have anti-tumor activity (Tavares-Murta et al., 2010). There are various factors for checking the status of immune system. NLR is a simple and effective marker of inflammation and is calculated by dividing the absolute peripheral blood neutrophils count by the absolute lymphocytes count.

NLR has been used as a prognostic factor in various types of cancers and also for examining the immune system (Guthrie et al., 2013). In a study on the relationship between NLR and CIN in South Korea, it was found that individuals with a high NLR level had a high risk of recurrence (Chun et al., 2017).

The aims of the current study were i) to examine the relationship between NLR and recurrence rate in CIN patients and ii) to investigate RFS and its prognostic factors in such patients.

\section{Materials and Methods}

The present study is a historical cohort study on a population of patients who received excisional treatment due to CIN positive pathology at the Department of Oncology, Imam Hossein Hospital, Tehran, Iran from 2009 to 2017 and followed-up until January 2018. The excisional procedure was performed as a LEEP or CKC by a skilled gynecologist. Demographic, clinical, pathological data, and hematological findings of these patients were extracted from their records. While complete blood count $(\mathrm{CBC})$ tests were taken routinely before surgery, the pre-operative $\mathrm{CBC}$ of these patients were recorded. The NLR index was calculated using neutrophil and lymphocyte counts reported in CBC prior to surgery. Patients with incomplete medical information and CBC during admission, complete absence of patient follow-up and related pathology files, previous history of topical or systemic infection, infection at the time of admission, and bone marrow suppression or taking immunosuppressive drugs were excluded from this study.

Eligible individuals were enrolled in this study and patients' follow-ups were examined using the record of hospital's clinics and its Pathology Department. Pathologic studies were carried out by a pathologist in the pathology center. Due to a high HPV test cost in our country and patients' financial inability, our follow-up protocol was carried out as colposcopy at 6- and 12-months intervals, and then annually. The recurrence was considered by histopathology, CIN I, CIN II and CIN III reports. The results are based on histopathology findings where the presence of mitotic forms and immature cells in the lower, middle and upper thirds of the epithelium were considered as CIN I, CIN II and CIN III, respectively. In the absence of recurrence, the follow-up period was considered as treatment interval until the last visit. In case of hysterectomy, the history of hysterectomy was considered as the end of follow-up. During this period, 514 individuals were treated using excisional procedure at the hospital's clinic center from 2009 to 2017. Of these, 14 individuals were excluded due to impairment of immunity and underlying illnesses. Another 193 patients were also excluded due to the lack of follow-up.

The sample size was calculated to detect the hazard ratio of 3.54 for the risk of recurrence of NLR 2.1 (Chun et al., 2017) with power of $80 \%$, alpha of 0.05 , and attrition rate of 10 percent. The required sample size was calculated to be at least 301 patients. In total, the information of 307 patients were assessed (Figure 1). Written informed consent was obtained from all eligible patients. This study was approved by the ethic committee of Shahid Beheshti University of Medical Sciences (IR.SBMU.RETECH. 1397.1360)

\section{Statistical method}

Quantitative data are presented as mean and standard deviations and qualitative data are presented as frequency and percentage. The diagnostic value of NLR in the prediction of recurrence was determined using a rock curve and was generated by the Youden index. The generated NLR value was 1.9. The sensitivity and specificity indices were calculated for the estimation of the cumulative point. Based on the cut-off point, patients were divided into low $(\mathrm{NLR}<1.9)$ and high NLR $(\mathrm{NLR} \geq 1.9)$ level groups. The demographic and clinical characteristics of the patients were evaluated based on T-independent and Chi-square tests. Logistic regression analysis was used to evaluate the factors affecting the recurrence. The effect of NLR on RFS was evaluated using Kaplan-Meier curve. Cox regression was used to evaluate the factors affecting the time to recurrence. Statistical tests were performed at a significant level of 0.05 and were two-tailed. The $\mathrm{R}$ package version 3.2.1 was used for data analysis.

\section{Results}

In this study, 307 women diagnosed with CIN were examined. The mean age of patients was 40.36 years with a standard deviation of 9.14. The mean NLR of patients was $1.78 \pm 0.953$ prior to excisional procedure. The mean size of excised tissue during excision was $2.35 \pm 0.645$ $\mathrm{cm}^{2}$. The clinical information of patients is presented in Table 1. The recurrence was observed among 38 $(12.4 \%)$ patients. The area under the receiver operating characteristic (ROC) curve for diagnosis of recurrence was remarkable (AUC $=0.801 ; 95 \% \mathrm{CI}$ of $(0.723,0.879)$; $\mathrm{p}<0.001$ ) (Figure 2). The NLR cut-off point of 1.9 was 
Table 1. Description and Comparison of Clinicopathologic and Laboratory Parameters of Patients According to the NLR Level

\begin{tabular}{|c|c|c|c|c|c|}
\hline Variables & & Total $(n=307)$ & NLR $<1.9(n=209)$ & NLR $(n=98)$ & $\mathrm{P}$ value \\
\hline Age & & $40.36 \pm 9.14$ & $40.64 \pm 9.41$ & $39.76 \pm 8.54$ & 0.429 \\
\hline Excised tissue $\left(\mathrm{cm}^{2}\right)$ & & $2.35 \pm 0.645$ & $2.39 \pm 0.625$ & $2.25 \pm 0.680$ & 0.085 \\
\hline \multirow[t]{2}{*}{ Delivery } & $<3$ & $170(55.4)$ & $115(55)$ & $55(56.1)$ & 0.857 \\
\hline & 3 & $137(44.6)$ & $94(45)$ & $43(43.9)$ & \\
\hline \multirow[t]{2}{*}{ Delivery type } & NVD & $228(74.3)$ & $156(74.6)$ & $72(73.5)$ & 0.323 \\
\hline & $\mathrm{CS}$ & $60(19.5)$ & $45(21.5)$ & $15(15.3)$ & \\
\hline \multirow[t]{2}{*}{ Smoke } & yes & $11(3.6)$ & $5(2.4)$ & $6(6.1)$ & 0.111 \\
\hline & no & $296(96.4)$ & $204(97.6)$ & $92(93.9)$ & \\
\hline \multirow[t]{3}{*}{ Contraception } & nothing & $36(11.7)$ & $26(12.4)$ & $10(10.2)$ & 0.093 \\
\hline & OCP & $65(21.2)$ & $37(17.7)$ & $28(28.6)$ & \\
\hline & others & $206(67.1)$ & $146(69.9)$ & $60(61.2)$ & \\
\hline \multirow[t]{2}{*}{ History of genital wart } & No & $250(81.4)$ & $174(83.3)$ & $76(77.6)$ & 0.231 \\
\hline & yes & $57(18.6)$ & $35(16.7)$ & $22(22.4)$ & \\
\hline \multirow[t]{2}{*}{ History of genital herpes } & No & $303(98.7)$ & 207 (99) & $96(98)$ & 0.595 \\
\hline & yes & $4(1.3)$ & $2(1)$ & $2(2)$ & \\
\hline \multirow[t]{6}{*}{ Pap smear } & Normal & $129(42)$ & $91(43.5)$ & $38(38.8)$ & 0.73 \\
\hline & LSIL & $34(11.1)$ & $24(11.5)$ & $10(10.2)$ & \\
\hline & HSIL & $22(7.2)$ & $16(7.7)$ & $6(6.1)$ & \\
\hline & ASCUC & $65(21.2)$ & $44(21.1)$ & $21(21.4)$ & \\
\hline & Inflammatory & $22(7.2)$ & $14(6.7)$ & $8(8.2)$ & \\
\hline & Unknown & $35(11.4)$ & $20(9.6)$ & $15(15.3)$ & \\
\hline \multirow[t]{3}{*}{ Treatment cause } & CIN I & $46(15)$ & $28(13.4)$ & $18(18.4)$ & 0.516 \\
\hline & CIN II & $204(66.4)$ & $142(67.9)$ & $62(63.3)$ & \\
\hline & CIN III & $57(18.6)$ & $39(18.7)$ & $18(18.4)$ & \\
\hline \multirow[t]{2}{*}{ Treatment type } & LEEP & $235(76.5)$ & $154(73.7)$ & $81(82.7)$ & 0.084 \\
\hline & Cold knife conization & $72(23.5)$ & $55(26.3)$ & $17(17.3)$ & \\
\hline \multirow[t]{2}{*}{ Margin } & involved & $28(9.1)$ & $14(6.7)$ & $14(14.3)$ & 0.031 \\
\hline & uninvolved & 279 (90.9) & $195(93.3)$ & $84(85.7)$ & \\
\hline \multirow[t]{4}{*}{ Recurrence type } & CIN I & $18(47.4)$ & $9(90)$ & $9(32.1)$ & 0.019 \\
\hline & CIN II & $17(44.7)$ & $1(10)$ & $16(57.1)$ & \\
\hline & CIN III & $2(5.3)$ & $0(0)$ & $2(7.1)$ & \\
\hline & Carcinoma in situ & $1(2.6)$ & $0(0)$ & $1(3.6)$ & \\
\hline $\mathrm{Hb}$ & & $12.32 \pm 1.19$ & $12.31 \pm 1.13$ & $12.33 \pm 1.31$ & 0.882 \\
\hline $\mathrm{MCV}$ & & $84.71 \pm 8.50$ & $84.73 \pm 9.05$ & $84.67 \pm 7.25$ & 0.956 \\
\hline $\mathrm{RBC}$ & & $4.37 \pm 0.473$ & $4.33 \pm 0.471$ & $4.46 \pm 0.467$ & 0.023 \\
\hline PLT & & $260.16 \pm 64.65$ & $261.8 \pm 61.3$ & $256.5 \pm 71.43$ & 0.509 \\
\hline WBC & & $7263.8 \pm 1842.7$ & $7093.3 \pm 1691.7$ & $7627.6 \pm 2092.6$ & 0.018 \\
\hline ANC & & $4111.1 \pm 1389.5$ & $3738 \pm 1112.7$ & $4906.6 \pm 1580.1$ & $<0.001$ \\
\hline AMC & & $375.4 \pm 200.9$ & $377.1 \pm 212.9$ & $371.9 \pm 173.5$ & 0.833 \\
\hline $\mathrm{AEC}$ & & $183.44 \pm 136.3$ & $186.4 \pm 141.7$ & $177.1 \pm 124.5$ & 0.576 \\
\hline ALC & & $2510.3 \pm 725.9$ & $2744.8 \pm 686.7$ & $2012.7 \pm 531.8$ & $<0.001$ \\
\hline PLR & & $113.3 \pm 46.11$ & $101.72 \pm 36.38$ & $137.8 \pm 54.47$ & $<0.001$ \\
\hline LMR & & $9.68 \pm 11.14$ & $11.05 \pm 12.87$ & $6.88 \pm 5.37$ & $<0.001$ \\
\hline
\end{tabular}

Data are represented as frequency (percent) or mean \pm SD. NVD, natural vaginal delivery; C/S, cesarean section; OCP, oral contraceptive pill; LSIL, low grade squamous intraepithelial neoplasia; HSIL, high grade squamous intraepithelial neoplasia; ASCUS, atypical squamous cell undetermined significant; CIN, cervical intraepithelial neoplasia; LEEP, Loop Electrosurgical Excision Procedure; Hb, hemoglobin concentration; MCV, mean corpuscular volume; RBC, Red blood cell count; PLT, platelet; WBC, white blood cell count; ANC, absolute neutrophil count; AMC, absolute monocyte count; AEC, absolute eosinophil count; ALC, absolute lymphocyte count; PLR, platelet-lymphocyte ratio; LMR, lymphocyte-monocyte ratio; NLR, neutrophil-lymphocyte ratio. 
Table 2. Description and Assessment of Factors Affecting Recurrence of Disease

\begin{tabular}{|c|c|c|c|c|c|c|c|}
\hline \multirow[b]{2}{*}{ Variable } & & \multicolumn{2}{|c|}{ Recurrence } & \multicolumn{2}{|r|}{ unadjusted } & \multicolumn{2}{|r|}{ adjusted } \\
\hline & & No $(n=269)$ & Yes $(\mathrm{n}=38)$ & $\mathrm{P}$ value & OR $(95 \% \mathrm{CI})$ & $\mathrm{P}$ value & OR $(95 \% \mathrm{CI})$ \\
\hline Age & & $40.95 \pm 9.05$ & $36.18 \pm 8.79$ & 0.003 & $0.941(0.904-0.980)$ & 0.019 & $0.927(0.870-0.988)$ \\
\hline Excised tissue $\left(\mathrm{cm}^{2}\right)$ & & $2.45 \pm 0.0601$ & $1.63 \pm 0.476$ & $<0.001$ & $0.067(0.028-0.158)$ & $<0.001$ & $0.041(0.013-0.135)$ \\
\hline \multirow[t]{2}{*}{ Delivery number } & $<3$ & $142(83.5)$ & $28(16.51)$ & 0.015 & $2.50(1.17-5.36)$ & 0.001 & $7.01(2.16-22.76)$ \\
\hline & 3 & $127(92.7)$ & $10(7.3)$ & & - & - & \\
\hline \multirow[t]{3}{*}{ Delivery type } & non & $15(78.9)$ & $4(21.1)$ & & - & & \\
\hline & NVD & 203 (89) & $25(11)$ & 0.199 & $0.462(0.142-1.50)$ & & \\
\hline & $\mathrm{CS}$ & $51(85)$ & $9(15)$ & 0.537 & $0.662(0.178-2.46)$ & & \\
\hline \multirow[t]{2}{*}{ Smoke } & no & $261(88.2)$ & $35(11.8)$ & & - & & \\
\hline & yes & $8(72.7)$ & $3(27.3)$ & 0.142 & $2.80(0.708-11.04)$ & & \\
\hline \multirow[t]{3}{*}{ Contraception } & nothing & $32(88.9)$ & $4(11.1)$ & & - & & \\
\hline & $\mathrm{OCP}$ & $56(86.2)$ & $9(13.8)$ & 0.695 & $1.29(0.366-4.51)$ & & \\
\hline & others & $181(87.9)$ & $25(12.1)$ & 0.861 & $1.11(0.360-3.39)$ & & \\
\hline \multirow[t]{2}{*}{ History of genital wart } & No & $223(89.2)$ & $27(10.8)$ & & - & & \\
\hline & yes & $46(80.7)$ & $11(19.3)$ & 0.083 & $1.98(0.915-4.26)$ & & \\
\hline \multirow[t]{2}{*}{ History of genital herpes } & No & $267(88.1)$ & $36(11.9)$ & & - & & - \\
\hline & yes & $2(50)$ & $2(50)$ & 0.049 & $7.42(1.01-54.29)$ & 0.104 & $23.16(0.527-1017)$ \\
\hline \multirow[t]{6}{*}{ Pap smear } & Normal & $116(89.9)$ & $13(10.1)$ & & - & & \\
\hline & LSIL & $32(94.1)$ & $2(5.9)$ & 0.457 & $0.558(0.120-2.60)$ & & \\
\hline & HSIL & $17(77.3)$ & $5(22.7)$ & 0.1 & $2.62(0.831-8.29)$ & & \\
\hline & ASCUC & $54(83.1)$ & $11(16.9)$ & 0.176 & $1.82(0.765-4.32)$ & & \\
\hline & Inflammatory & $18(81.8)$ & $4(18.2)$ & 0.274 & $1.98(0.582-6.755)$ & & \\
\hline & Unknown & $32(91.4)$ & $3(8.6)$ & 0.79 & $0.837(0.225-3.12)$ & & \\
\hline \multirow[t]{3}{*}{ Treatment cause } & CIN I & $43(93.5)$ & $3(6.5)$ & - & & & \\
\hline & CIN II & $177(86.8)$ & $27(13.2)$ & 0.216 & $2.19(0.634-7.54)$ & & \\
\hline & CIN III & $49(86)$ & $8(14)$ & 0.23 & $2.34(0.584-9.38)$ & & \\
\hline \multirow[t]{2}{*}{ Treatment type } & Cold knife conization & $67(93.1)$ & $5(6.9)$ & 0.117 & - & & \\
\hline & LEEP & $202(86)$ & $33(14)$ & & $2.19(0.821-5.84)$ & & \\
\hline \multirow[t]{2}{*}{ Margin } & uninvolved & $251(90)$ & $28(10)$ & & - & & - \\
\hline & involved & $18(64.3)$ & $10(35.7)$ & $<0.001$ & $4.98(2.09-11.84)$ & 0.013 & $6.70(1.49-30.04))$ \\
\hline \multirow[t]{2}{*}{ NLR } & $<1.9$ & $199(95.2)$ & $10(4.8)$ & $<0.001$ & - & $<0.001$ & - \\
\hline & $\geq 1.9$ & $70(71.4)$ & $28(28.6)$ & & $7.96(3.68-17.22)$ & & $8.49(2.78-25.93)$ \\
\hline $\mathrm{Hb}$ & & $12.36 \pm 1.14$ & $12.04 \pm 1.45$ & 0.132 & $0.806(0.608-1.07)$ & & \\
\hline $\mathrm{MCV}$ & & $84.99 \pm 7.44$ & $82.73 \pm 13.87$ & 0.148 & $0.978(0.948-1.01)$ & & \\
\hline $\mathrm{RBC}$ & & $4.38 \pm 0.477$ & $4.34 \pm 0.0451$ & 0.623 & $0.830(0.395-1.75)$ & & \\
\hline PLT & & $261.9 \pm 63.89$ & $247.3 \pm 69.27$ & 0.189 & $0.996(0.990-1.00)$ & & \\
\hline WBC & & $7168.4 \pm 1660.5$ & $7939.5 \pm 2752.5$ & 0.017 & $1.24(1.04-1.47)$ & 0.539 & $1.59(0.364-6.91)$ \\
\hline ANC & & $3998.2 \pm 1192.9$ & $4909.7 \pm 2216.2$ & $<0.001$ & $1.52(1.20-1.91)$ & 0.816 & $1.18(0.278-5.05)$ \\
\hline $\mathrm{AMC}$ & & $373.4 \pm 205.2$ & $389.7 \pm 169.5$ & 0.641 & $1.48(0.284-7.73)$ & & \\
\hline $\mathrm{AEC}$ & & $184 \pm 141.1$ & $179.3 \pm 97.08$ & 0.841 & $1(0.997-1.002)$ & & \\
\hline ALC & & $2579.6 \pm 702.2$ & $2021.6 \pm 711.9$ & $<0.001$ & $0.290(0.164-0.513)$ & 0.005 & $0.044(0.005-0.387)$ \\
\hline PLR & & $110.1 \pm 43.25$ & $135.9 \pm 58.5$ & 0.002 & $1.01(0.003-1.02)$ & 0.183 & $0.989(0.973-1.01)$ \\
\hline LMR & & $10.13 \pm 11.73$ & $6.52 \pm 4.33$ & 0.027 & $0.896(0.814-0.988)$ & 0.643 & $0.967(0.838-1.12)$ \\
\hline
\end{tabular}

Data are represented as mean \pm SD or frequency (percent) whenever it is needed. NVD, natural vaginal delivery; C/S, cesarean section; OCP, oral contraceptive pill; LSIL, low grade squamous intraepithelial neoplasia; HSIL, high grade squamous intraepithelial neoplasia; ASCUS, atypical squamous cell undetermined significant; CIN, cervical intraepithelial neoplasia; LEEP, Loop Electrosurgical Excision Procedure; Hb, hemoglobin concentration; MCV, mean corpuscular volume; RBC, Red blood cell count; PLT, platelet; WBC, white blood cell count; ANC, absolute neutrophil count; AMC, absolute monocyte count; AEC, absolute eosinophil count; ALC, absolute lymphocyte count; PLR, platelet-lymphocyte ratio; LMR, lymphocyte-monocyte ratio; NLR, neutrophil-lymphocyte ratio.

obtained using the Youden index. The sensitivity and specificity of the NLR cut-off point in the diagnosis of recurrence were $\operatorname{Sen}=0.737 ; 95 \% \mathrm{CI}(0.569 .0 .866)$ and $\mathrm{SP}=0.739 ; 95 \%$ CI $(0.682 .79)$, respectively. The NLR level was less than 1.9 in 209 (68.1\%) patients and was greater than or equal to 1.9 in $98(31.9 \%)$ patients. The recurrence rate in patients with $\mathrm{NLR}<1.9$ and $\mathrm{NLR} \geq 1.9$ was $4.8 \%$ and $28.6 \%$, respectively. The odds of recurrence was higher in patients with $\mathrm{NLR} \geq 1.9(\mathrm{OR}=7.96 ; 95 \% \mathrm{CI}$ $3.68,17.22)$ ) (Figure 3). In the low NLR group, 9 (90\%) 


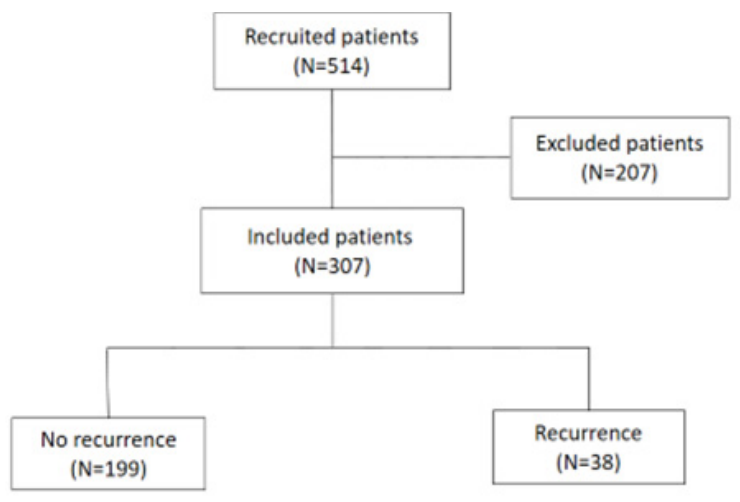

Figure 1. Diagram of Participant Recruitment

patients had CIN I and $1(10 \%)$ had CIN II. But in the high NLR group, the recurrence of CIN I was observed in 9 (32.1\%), CIN II in 16 (57.1\%), CIN III in $2(7.7 \%)$ patients and carcinoma in situ in $1(6.3 \%)$ patient. CIN I was significantly more common in the low NLR group than in

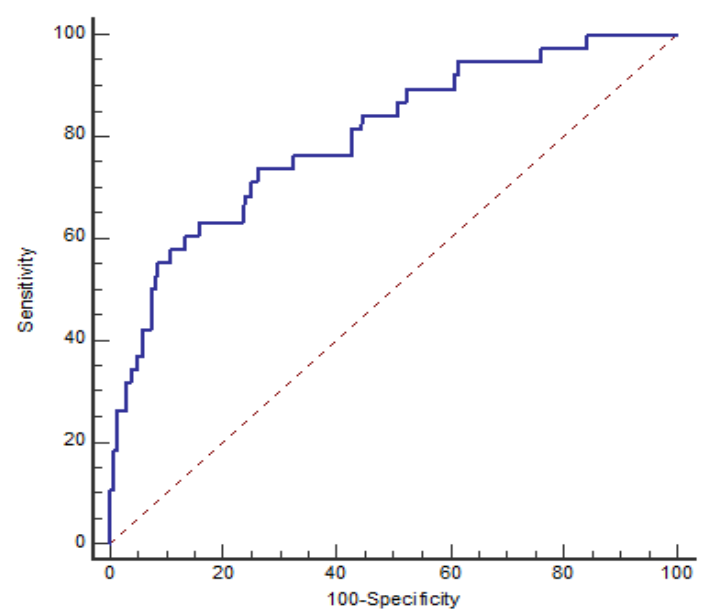

Figure 2. Receiver Operating Characteristics (ROC) Curve of NLR for Predicting Recurrence. NLR, Neutrophil-Lymphocyte Ratio

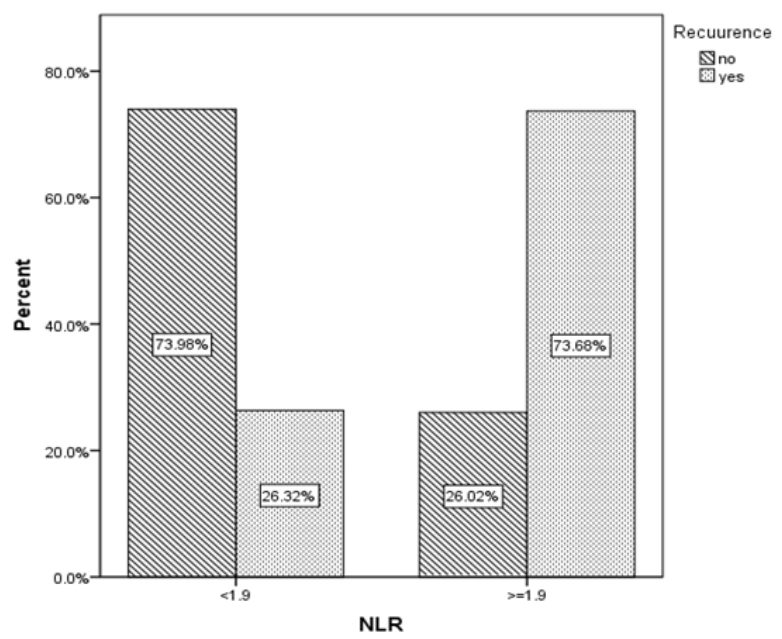

Figure 3. Recurrence Rate According to the NLR Level. NLR, Neutrophil-Lymphocyte Ratio

the high NLR one $(p=0.019)$. It was also observed that even after excluding patients with CIN I, the NLR levels were still associated with the recurrence, as in patients with lower NLRs, the recurrence rate was significantly lower than in other patients ( $5 \%$ vs. $32.5 \%$; $<0.001)$. The comparison between demographic and clinical parameters of these patients based on the NLR level is presented in Table 1. There was not any significant difference between these groups regarding the type of delivery $(p=0.323)$. The proportion of patients with marginal involvement in the high NLR group was significantly higher than in the low NLR one $(14.3 \%$ vs. $6.7 \%$; $=0.031)$.

In univariate analysis, factors that increase the risk of recurrence were: younger age $(p<0.001)$, less than three deliveries $(p=0.015)$, the incidence of genital herpes $(p=0.049)$, marginal involvement $(p<0.001)$, lower ANC $(\mathrm{p}<0.001)$, ALC $(\mathrm{p}<0.001)$ and Lymphocyte Monocytic Ratio (LMR) $(p=0.027)$ levels, and higher Platelet (PLT) levels $(p=0.002)$. Furthermore, the odds of recurrence in patients treated with LEEP method was two times

Table 3. Result of Univariate Cox Models and Multivariate Cox Model to Determine Factors Affecting RFS

\begin{tabular}{llcccc}
\hline Variable & & Crude hazard ration & P value & Adjusted hazard ration & P value \\
\hline Age & & $0.949(0.915-0.984)$ & 0.005 & & 0.032 \\
Excised tissue & $0.090(0.044-0.181)$ & $<0.001$ & $0.325(0.136-0.912)$ & \\
Smoking & yes vs.no & $3.13(0.958-10.19)$ & 0.059 & & $<0.001$ \\
History of genital wart & yes vs.no & $2.04(1.01-4.09))$ & 0.049 & & 0.001 \\
History of genital herpes & yes vs.no & $5.58(1.34-23.20)$ & 0.018 & $16.49(3.06-88.82)$ & 0.01 \\
margin & Involved vs. uninvolved & $4.12(2.01-8.50)$ & $<0.001$ & $4.52(1.89-10.84)$ & $<0.001$ \\
Delivery number & $<3$ vs. $\geq 3$ & $2.30(1.12-4.73)$ & 0.024 & $2.88(1.29-6.47)$ & 0.017 \\
NLR & $\geq 1.9$ vs. $<1.9$ & $6.48(3.15-13.34)$ & $<0.001$ & $4.55(1.97-10.51)$ & \\
RBC & & $0.778(0.602-1.01)$ & 0.056 & & $1.27(1.04-1.55)$ \\
WBC & $1.22(1.05-1.42)$ & 0.01 & & $<0.001$ \\
ANC & $1.41(1.20-1.67)$ & $<0.001$ & & $0.258(0.121-0.549)$ \\
ALC & & $0.337(0.203-0.558)$ & $<0.001$ & & \\
PLR & & $1.01(1.003-1.012)$ & 0.002 & & \\
LMR & $0.893(0.812-0.981)$ & 0.019 & & \\
\hline
\end{tabular}

RBC, Red blood cell count; WBC, white blood cell count; ANC, absolute neutrophil count; ALC, absolute lymphocyte count; PLR, plateletlymphocyte ratio; LMR, lymphocyte-monocyte ratio; NLR, neutrophil-lymphocyte ratio 


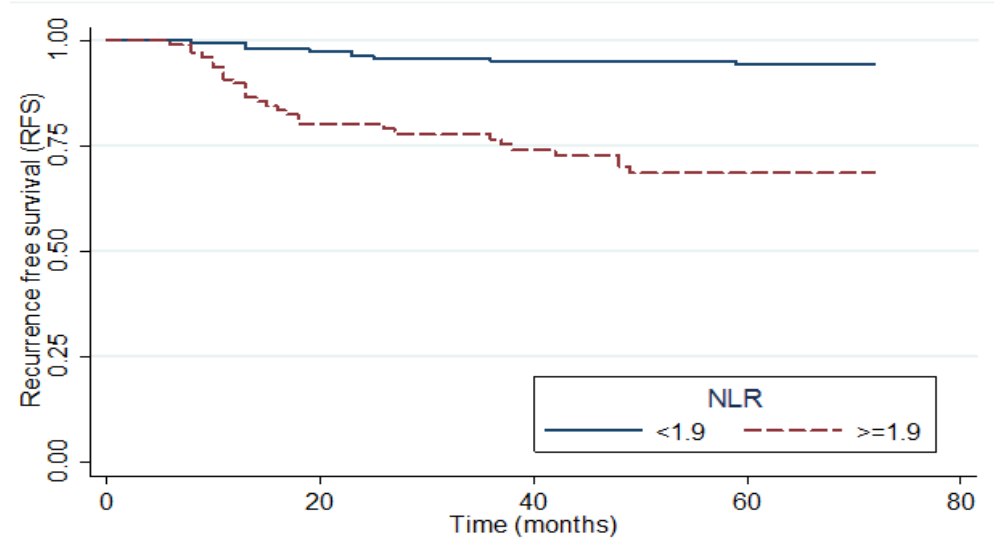

Figure 4. Kaplan-Mire Plot of RFS According to the NLR Level

higher than in patients treated with cold knife conization method, but this difference was not statistically significant $(\mathrm{OR}=2.19 ; \mathrm{p}=0.117)$. According to multiple logistic regression results, the odds of recurrence in patients with the NLR level higher than 1.9 was approximately 8.5 times higher than in other patients $(\mathrm{OR}=8.49(2.78-25.93))$. The risk of recurrence was reduced by $7 \%(\mathrm{OR}=0.927$ (0.988-0.870)) for a one-year increase in age. Increase in tissue size during excisional treatment reduced the chance of recurrence $(\mathrm{OR}=0.041(0.013-0.135))$ (Table 2 ). The risk of recurrence in women with a maximum of two deliveries was about 7 times higher than in other women $(\mathrm{OR}=7.01(2.16-22.76))($ Table 2$)$. The incidence of recurrence in women with marginal involvement was about 6.7 times higher than in women without marginal involvement $(\mathrm{OR}=6.70(1.49-30.04))$, and ALC significantly increased the risk of recurrence $(\mathrm{OR}=0.044$ (0.0087-0.387) (Table 2).

The median follow-up time was 60 months with minimum and maximum follow-ups of 12 and 72 months, respectively. The median RFS for patients was 48 months. RFS was significantly higher in the low NLR group than in the high NLR one $(\mathrm{P}<0.001)$. Figure 4 shows RFS Kaplan-Meier plot based on NLR level. In univariate analysis, less number of deliveries $(\mathrm{P}=0.024)$, marginal involvement $(\mathrm{P}<0.001)$, smoking $(\mathrm{P}=0.047)$, the history of genital wart $(\mathrm{P}=0.049)$ and genital herpes $(\mathrm{P}=0.018)$, smaller tissue size during excisional treatment $(\mathrm{P}<0.001)$, younger age $(\mathrm{P}=0.005)$, lower red blood cell $(\mathrm{RBC})$ count $(\mathrm{P}=0.056), \operatorname{ALC}(\mathrm{P}<0.001)$ and LMR $(\mathrm{P}=0.019)$, and higher total WBC count $(\mathrm{P}=0.010)$, ANC $(\mathrm{P}<0.001)$ and $\operatorname{PLR}(\mathrm{P}=0.002)$ were associated with reduction in the time to recurrence. Also, the results of Cox regression showed that increase in the size of excised tissue during excision $(\mathrm{HR}=0.325 ; 95 \% \mathrm{CI}(0.936-0.136))$ and lower ALC (HR $=0.258 ; 95 \% \mathrm{CI}(0.121-0.549))$ reduced the hazard of recurrence. Furthermore, a history of herpes disease (HR $=16.49 ; 95 \% \mathrm{CI}(3.06-88.82))$, marginal involvement $(\mathrm{HR}$ $=4.52 ; 95 \% \mathrm{CI}(1.89-10.84))$, less deliveries $(\mathrm{HR}=2.88$; 95\% CI (1.29)), higher NLR $(\mathrm{HR}=4.55 ; 95 \% \mathrm{CI})$ (1.97-10.51) and total WBC count $(\mathrm{HR}=1.27 ; 95 \% \mathrm{CI}$ (1.04-1.55)) levels, significantly increased the hazard of recurrence (Table 3 ).

\section{Discussion}

In the current study, NLR and total WBC count were known to be prognostic factors for predicting the likelihood of recurrence in patients who underwent excisional procedure for CIN. Chun et al., (2017) investigated the relationship between NLR and recurrence of CIN in Korea. They studied 230 patients who underwent excisional procedure for CIN. The patients were divided into two group: low (63 patients) and high (167 patients) NLR groups. They found that NLR is a significant prognostic factor for RFS in patients with CIN (Chun et al., 2017). In our study, the NLR cut off point was 1.9 (generated by the Youden Index) which was found to be more sensitive and specific than the cutoff used by Chun et al., (2017) and we also had a larger sample size compared to their study.

In another study conducted by Misunuma et al., (2016) on 56 patients with cervical cancer, NLR was found to be a prognostic factor for overall survival (OS) and progression free survival (PFS) in patients. Patients with high NLR had shorter OS and PFS. The same results were found in our study. Several studies have been conducted on the relation of NLR as a prognostic factor with various types of cancers. Studies on different cancers such as colorectal cancer, squamous cell carcinoma of lung and thymoma revealed that high NLR levels prior to surgery were associated with worse survival, and patients with a higher NLR level had a shorter disease free survival (DFS) (Walsh et al., 2005; Sarraf et al., 2009; Muriana et al., 2018). The same findings were also mirrored in our study.

We found that in univariate cox analysis patients with shorter RFS had higher ANC. The same results were also reported in other studies on the immune system changes related to different cancers such as pancreas, localized papillary renal cell carcinoma and metastatic melanoma. It has been shown that neutrophilia can be a prognostic factor for predicting the survival of patients, and neutrophilia has been associated with a reduction in survival (Schmidt et al., 2005; Huang et al., 2015; Xiao et al., 2016). We also found that in univariate cox analysis, ANC was higher in patients with shorter RFS.

According to our results, patients with a higher PLR level had a higher recurrence rate. Onal et al., (2016) 
evaluated NLR and PLR as prognostic factors in patients with cervical cancer prior to definitive chemoradiation therapy (ChRT) and found that NLR and PLR were associated with OS and RFS. OS and RFS were shorter in patients with higher NLR and PLR levels. In other studies, a higher PLR level in preoperative phase resulted in a worse oncological outcome and a higher recurrence rate in patients with prostate and epithelial ovarian cancers (Raungkaewmanee et al., 2012; Guo et al., 2018). In our univariate analysis, patients with higher PLR and ANC levels showed a higher recurrence rate but in multivariate analysis, there was not any statistically significant relationship between PLR and RFS. Therefore, it can be concluded that NLR and total WBC count were more predictive factors.

Based on our results, NLR might be similar to well-known biomarkers in decision making for the treatment of patients with low and intermediate grade pathologies. NLR with a high index can serve as a potentially useful tool when selecting the type of treatment. Several biomarkers including p16, Ki 63 and P16INK4a have been used in various studies to identify management of CIN lesions(Vitale et al., 2016; Zhang et al., 2018). Zhang et al., (2018) showed that these biomarkers can increase the sensitivity of detecting CIN $2+$ lesions in patients with ASCUS and LSIL. It is worth noting that NLR is an inexpensive, convenient and widely accessible marker.

We also found that there was a higher recurrence rate in patients with a positive margin. This finding is in consistent with the results of Serati et al., (2012) and Lodi et al., (2011) on CIN recurrence-associated risk factors. But it is important to note that according to our center's protocol, reconization surgery plan is always performed for patients with positive margins. Finally, patients with free margins are entered into the follow-up phase. Despite performing reconization surgery, patients with positive margins still had a higher recurrence rate in the initial surgery. This might counteract the protective effects of reconization against the recurrence in positive margin treatment with excisional procedure. According to our result, reconization surgery had no effects on the reduction of recurrence. Moreover, this surgery has emotional side effects. Therefore, it could be concluded that determining prognostic factors prior to the surgery and performing a complete surgery based on these factors is a way of avoiding reconization.

The history of genital herpes infection is another recurrence-associated factor which may increase the likelihood of recurrence. Considering the genital herpes as a sexual transmitted disease (STD), the probability of high-risk behaviors and infection with persistent high-risk HPV can be higher in people with a history of genital herpes. Persistent high-risk HPV is one of the known factors that increases the recurrence of CIN.

We also found that the size of excised tissue during initial excisional surgery was associated with the recurrence rate and RFS which is inconsistent with the results of Maria Kolben et al., (2018). They reported that the size of excised tissue during excision was not associated with the rate of recurrence. This difference might be due to a longer follow-up period in our study. It seems that increase in the tissue size decreased the rate of recurrence. Due to the multifocality nature of CIN, removing larger samples during surgery is accompanied by skip lesion which can decrease the likelihood of recurrence. But the likelihood of treatment-associated side effects such as cervical stenosis and pregnancy-related complications should be regarded, and the balance between two should be kept. In our study, information on the size of excised tissue during surgery and marginal involvement was available, but the lesion distance from margin and the amount of free margin were unknown, which was a weak point in our study. But evidence suggests that removing more tissue during surgery will reduce the chance of recurrence. Based on this finding, it is recommended that the free margin should be investigated and reported in pathological studies of CIN.

In conclusion, NLR can be used as a prognostic factor to predict the recurrence of $\mathrm{CIN}$ after excisional treatment. Accordingly, in patients with high NLR levels in the preoperative phase, we can remove a larger tissue during surgery, or have short-interval follow-ups on patients and make them aware of the high risk of recurrence. Hematologic markers evaluation is not only reliable but also simple and inexpensive method in the prediction of recurrence. Based on the results of other studies and the findings of our study, it is possible to determine the NLR cut-off which might be used for patients with CIN II and the type of treatment should be precisely determined in CIN II patients with NLR level higher than cut-off point. It can be used as a biomarker for young patients and those with the possible impact of cervical treatment on their subsequent fertility and pregnancy and to detect patients with a higher likelihood of recurrence and to avoid possible side effects in low-risk individuals. However, more studies are needed to make a definitive decision.

Our study is a retrospective study which is a weak points. To confirm these results, further studies should be conducted prospectively and with a larger sample size. Furthermore, it is recommended that future research focuses on understanding the relationship between total WBC count, ANC and PLR levels, and the recurrence rate in CIN patients.

\section{Acknowledgements}

The authors would like to thank the nursing, administrative and secretarial staff at the Obstetrics and Gynecology Department, Imam Hossein hospital for their contribution to the maintenance of our patients' records and the Preventative Gynecology Research Center for their support.

\section{Funding Statement}

This research was supported by Preventative Gynecology Research Center, Shahid Beheshti University of Medical Sciences.

\section{References}

Arbyn M, Redman CW, Verdoodt F, et al (2017). Incomplete Asian Pacific Journal of Cancer Prevention, Vol 202371 
excision of cervical precancer as a predictor of treatment failure: a systematic review and meta-analysis. Lancet Oncol, 18, 1665-79.

Ashrafganjoei T, Mohamadianamiri M, Farzaneh F, Hosseini MS, Arab M (2016). Investigating preoperative hematologic markers for prediction of ovarian cancer surgical outcome. Asian Pac J Cancer Prev, 17, 1445-8.

Bottazzi B, Riboli E, Mantovani A (2018). Aging, inflammation and cancer. Semin Immunol, 40, 74-82.

Chen J-y, Wang Z-1, Wang Z-y, Yang X-s (2018). The risk factors of residual lesions and recurrence of the high-grade cervical intraepithelial lesions (HSIL) patients with positive-margin after conization. Medicine (Baltimore), 97, e12792.

Chun S, Shin K, Kim KH, et al (2017). The neutrophil-lymphocyte ratio predicts recurrence of ervical intraepithelial Neoplasia. J Cancer, 8, 2205-11.

Garcia F, Hatch KD, Berek JS (2012). Berek and Novak's Gynecology, 15th ed. Intraepithelial Disease of the Cervix, Vagina, and Vulva. BerekJS, editor, Philadelphia: Lippincott Williams and Wilkins, pp 574-618.

Guo J, Fang J, Huang X, et al (2018). Prognostic role of neutrophil to lymphocyte ratio and platelet to lymphocyte ratio in prostate cancer: a meta-analysis of results from multivariate analysis. Int J Surg, 60, 216-23.

Guthrie GJ, Charles KA, Roxburgh CS, et al (2013). The systemic inflammation-based neutrophil-lymphocyte ratio: experience in patients with cancer. Crit Rev Oncol Hematol, $\mathbf{8 8}, 218-30$.

Huang J, Dahl DM, Huang J, et al (2015). Preoperative neutrophil-to-lymphocyte ratio and neutrophilia are independent predictors of recurrence in patients with localized papillary renal cell carcinoma. Biomed Res Int, 2015, 891045.

Lodi CT, Michelin MA, Lima MI, et al (2011). Factors associated with recurrence of cervical intraepithelial neoplasia after conization in HIV-infected and noninfected women. Arch Gynecol Obstet, 284, 191-7.

Maria Kolben T, T Etzel L, Bergauer F, et al (2019). A randomized trial comparing limited-excision conisation to Large Loop Excision of the Transformation Zone (LLETZ) in cervical dysplasia patients. J Gynecol Oncol, 30, e42.

Martin-Hirsch PP, Paraskevaidis E, Kitchener HCs (2000). Surgery for cervical intraepithelial neoplasia. Cochrane Database Syst Rev, 2, CD001318.

Mizunuma M, Yokoyama Y, Futagami M, et al (2015). The pretreatment neutrophil-to-lymphocyte ratio predicts therapeutic response to radiation therapy and concurrent chemoradiation therapy in uterine cervical cancer. Int J Clin Oncol, 20, 989-96.

Muriana P, Carretta A, Ciriaco P, Bandiera A, Negri G (2018). Assessment of the prognostic role of neutrophil-to-lymphocyte ratio following complete resection of thymoma. J Cardiothorac Surg, 13, 119.

Onal C, Guler OC, Yildirim BA (2016). Prognostic use of pretreatment hematologic parameters in patients receiving definitive chemoradiotherapy for cervical cancer. Int $J$ Gynecol Cancer, 26, 1169-1175.

Pesic M, Greten FR (2016). Inflammation and cancer: tissue regeneration gone awry. Curr Opin Cell Biol, 43, 55-61.

Raungkaewmanee S, Tangjitgamol S, Manusirivithaya S, Srijaipracharoen S, Thavaramara T (2012). Platelet to lymphocyte ratio as a prognostic factor for epithelial ovarian cancer. J Gynecol Oncol, 23, 265-73.

Rock JA, Jones HW (2016). cervical cancer precursors and their management. Te Linde's Operative Gynecology. 11 ed, Wolters Kluwer Health/Lippincott Williams and Wilkins, pp ,1179-92.
Sarraf KM, Belcher E, Raevsky E, et al (2009). Neutrophil/ lymphocyte ratio and its association with survival after complete resection in non-small cell lung cancer. $J$ Thorac Cardiovasc Surg, 137, 425-8.

Schmidt H, Bastholt L, Geertsen P, et al (2005). Elevated neutrophil and monocyte counts in peripheral blood are associated with poor survival in patients with metastatic melanoma: a prognostic model. Br J Cancer, 93, 273-8.

Serati M, Siesto G, Carollo S, et al (2012). Risk factors for cervical intraepithelial neoplasia recurrence after conization: a 10-year study. Eur J Obstet Gynecol Reprod Biol, 165, 86-90.

Tavares-Murta BM, Mendonça MA, Duarte NL, et al (2010). Systemic leukocyte alterations are associated with invasive uterine cervical cancer. Int J Gynecol Cancer, 20, 1154-9.

Vitale SG, Valenti G, Rapisarda AMC, et al (2016). P16INK4a as a progression/regression tumor marker in LSIL cervix lesions:our clinical experience. Eur J Gynecol Oncol, 37, 685-8.

Walsh S, Cook E, Goulder F, Justin T, Keeling N (2005). Neutrophil-lymphocyte ratio as a prognostic factor in colorectal cancer. J Surg Oncol, 91, 181-4.

Wright JD (2014). Cervical intraepithelial neoplasia: Terminology, incidence, pathogenesis and prevention. Lancet, 370, 1609-21.

Wright JD (2017). Cervical intraepithelial neoplasia: Treatment and follow-up. Post TW, ed. UpToDate. Waltham, MA: UpToDate Inc. https://www.uptodate.com (Accessed on 26 Nov, 2018.)

Xiao Y, Xie Z, Shao Z, et al (2016). Neutrophil and lymphocyte counts at diagnosis are associated with overall survival of pancreatic cancer: A retrospective cohort study. Medicine (Baltimore), 95, e5024.

Zhang R, Ge X, You K, et al (2018). P16/Ki 67 dual staining improves the detection specifity of high grade cervical lesions. J Obstet Gynecol Res, 44, 2077-84.

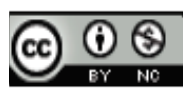

This work is licensed under a Creative Commons AttributionNon Commercial 4.0 International License. 